\title{
Combination treatment with VPA and MSCs-TRAIL could increase anti-tumor effects against intracranial glioma
}

\author{
SOON A. PARK ${ }^{1}$, HYE RIM HAN $^{1}$, STEPHEN AHN $^{2}$, CHUNG HEON RYU $^{3}$ and SIN-SOO JEUN ${ }^{1,2}$ \\ ${ }^{1}$ Department of Biomedicine and Health Sciences, College of Medicine; ${ }^{2}$ Department of Neurosurgery, \\ Seoul St. Mary's Hospital, The Catholic University of Korea, Seoul 06591; ${ }^{3}$ Department of \\ Clinical Laboratory Science, Daejeon Health Institute of Technology, Daejeon 34504, Republic of Korea
}

Received January 10, 2020; Accepted October 2, 2020

DOI: $10.3892 /$ or.2021.7937

\begin{abstract}
Human bone marrow-derived mesenchymal stem cells secreting tumor necrosis factor-related apoptosis-inducing ligand (MSCs-TRAIL) have demonstrated effective anti-tumor activity against various tumors including lung, pancreatic and prostate tumors, although several tumor types are not responsive. In such case, other reagents may decrease tumor growth via TRAIL-mediated cell death. The present study aimed to examine the effectiveness of valproic acid (VPA) in enhancing the efficacy of TRAIL, which was delivered using MSCs. Moreover, the present study examined the induced tumor tropism of MSCs via cell viability and migration assays. Combination treatment with VPA and MSCs-TRAIL enhanced the glioma therapeutic effect by increasing death receptor 5 and caspase activation. Migration assays identified increased MSC migration in VPA and MSCs-TRAIL-treated glioma cells and in the tumor site in glioma-bearing mice compared with VPA or MSC-TRAIL treatment alone. In vivo experiments demonstrated that MSC-based TRAIL gene delivery to VPA-treated tumors had greater therapeutic efficacy compared with treatment with each agent alone. These
\end{abstract}

Correspondence to: Professor Sin-Soo Jeun, Department of Neurosurgery, Seoul St. Mary's Hospital, The Catholic University of Korea, 222 Banpo-daero, Seocho-gu, Seoul 06591, Republic of Korea

E-mail: ssjeun@catholic.ac.kr

Professor Chung Heon Ryu, Department of Clinical Laboratory Science, Daejeon Health Institute of Technology, 21 Chung Jeong Street, Dong-gu, Daejeon 34504, Republic of Korea

E-mail: ryuch@hit.ac.kr

Abbreviations: VPA, valproic acid; TRAIL, tumor necrosis factor-related apoptosis-inducing ligand; MSCs-TRAIL, tumor necrosis factor-related apoptosis-inducing ligand-secreting human bone marrow-derived mesenchymal stem cells; CXCR4, C-X-C chemokine receptor type 4; SDF-1, stromal cell-derived factor-1; GBM, Glioblastoma multiforme

Key words: GBM, VPA, MSCs-TRAIL, CXCR4, SDF-1 findings suggested that VPA treatment increased the therapeutic efficacy of MSC-TRAIL via TRAIL-induced apoptosis and enhanced tropism of MSCs, which may offer a useful strategy for tumor gene therapy.

\section{Introduction}

Glioblastoma multiforme (GBM), the most common and aggressive malignant glioma type, has poor therapeutic outcomes, even after surgical resection, radiation and chemotherapy. After treatment using surgical resection, radiation and chemotherapy, the median survival of patients with GBM is 14.6 months for radiotherapy plus temozolomide and 12.1 months for radiotherapy alone (1). GBM can invade or infiltrate areas within or close to functional brain parenchyma (2), and novel strategies are required to improve anti-tumor effects on glioma to overcome these problems.

The tumor necrosis factor-related apoptosis-inducing ligand (TRAIL) triggers apoptosis in tumor cells without damaging healthy cells and tissues, and is used in various types of tumor therapies, including $\operatorname{GBM}(3,4)$. Human bone marrow-derived mesenchymal stem cells (hBM-MSCs; MSCs) have tumor-targeting characteristics and can be easily separated and engineered using viral vectors $(5,6)$. In addition, the migratory capacity of MSCs toward the tumor region increases chemokines or growth factors expressed and released by glioma cells, which mediates the tropism of MSCs $(7,8)$. To improve the secretion duration of TRAIL and deliver it to invaded tumor cells, the current study used MSCs (9-12).

TRAIL binds to death receptors (e.g. DR4 and DR5), which activates the caspase cascade (13). The loss of DRs increases the expression levels of caspase inhibitor, cellular FLICE-inhibitory protein and X-linked inhibitor of apoptosis protein, or causes alterations in the expression of the Bcl-2 protein family (13). TRAIL alone may be an insufficient to treat tumor, as multiple types of tumor cells, including most glioma cell, do not respond to TRAIL-induced apoptosis with TRAIL alone $(14,15)$. Previous studies have reported that chemotherapeutic agents or radiotherapy can enhance response to TRAIL by upregulating the TRAIL receptors in gliomas (16-18), suggesting that improved anti-tumor activity may be accomplished with combination treatments. 
Valproic acid (VPA) is a histone deacetylase inhibitor (HDACi) agent used in the treatment of epileptic seizures, bipolar disorders and migraines in clinical research (19-21). VPA exerts an anticancer effect; for instance, it increases the cytotoxicity effect to various cancer cell lines (22-24), as well as can induce apoptosis and growth arrest by activating the DR and caspase pathway (25-27). Therefore, the present study investigated the efficacy of adding VPA to MSCs secreting TRAIL (MSC-TRAIL) to increase the therapeutic effects of glioma treatment.

In the current study, it was hypothesized that VPA could enhance MSCs-TRAIL anticancer effect in glioma. To investigate a combined treatment of VPA and MSCs-TRAIL that enhances anticancer effects to glioma compared with each treatment alone, effect of combined treatment VPA and MSCs-TRAIL to glioma was examined using in vitro and in vivo models.

\section{Materials and methods}

Cell culture and reagents. All cells were maintained at $37^{\circ} \mathrm{C}$ in a 5\% $\mathrm{CO}_{2}$-humidified atmosphere. MSCs were obtained from the Catholic Institute of Cell Therapy. MSCs were grown in DMEM (Wisent, Inc.) supplemented with 20\% FBS (Wisent, Inc.), $10,000 \mu \mathrm{g} / \mathrm{ml}$ penicillin and streptomycin (Gibco; Thermo Fisher Scientific, Inc.), and were used for experiments during passages 3-5. A glioblastoma cell line of unknown origin (U87) and a glioblastoma cell line of U138 cells were purchased from the American Type Culture Collection (cat. nos. HTB-14 and HTB-16; https://web.expasy.org/cellosaurus/CVCL_0022) and cultured in DMEM (Wisent, Inc.) supplemented with 10\% FBS (Wisent, Inc.) and 10,000 $\mu \mathrm{g} / \mathrm{ml}$ penicillin and streptomycin (Gibco; Thermo Fisher Scientific, Inc.).

VPA was purchased from Sigma-Aldrich (Merck KGaA). Recombinant human TRAIL and DR5/Fc chimera proteins were acquired from R\&D Systems, Inc. DR5/Fc chimeric protein $(100 \mathrm{ng} / \mathrm{ml})$ was used at $37^{\circ} \mathrm{C}$ for $24 \mathrm{~h}$. Stromal cell-derived factor-1 (SDF-1) was purchased from Santa Cruz Biotechnology, Inc. The C-X-C chemokine receptor type 4 (CXCR4) antagonist AMD3100 was purchased from Sigma-Aldrich (Merck KGaA).

Adenoviral vectors and infection. The recombinant adenoviral vector encoding the gene for enhanced green fluorescent protein (Ad-GFP) and TRAIL (Ad-TRAIL) were engineered and provided using the Ad-Easy vector system, following the manufacturer's instructions [Quantum Biotechnologies (Pty) Ltd.]. Ad-GFP or Ad-TRAIL MSCs $\left(5 \times 10^{5}\right)$ were infected at a multiplicity of infection of 50 in OPTI-MEM serum free media for a $3 \mathrm{~h}$ incubation in $5 \% \mathrm{CO}_{2}$ humidified $37^{\circ} \mathrm{C}$ chamber. After 3 days, MSCs-TRAIL was used in the following experiments, as described previously (18).

Cytotoxic effect of VPA or TRAIL in glioma cells (co-culture). Cells were plated into wells of a 96-well plate at a density of $5 \times 10^{3}$ cells/well in medium containing $10 \%$ FBS and incubated at $37^{\circ} \mathrm{C}$ for $48 \mathrm{~h}$ in a $5 \% \mathrm{CO}_{2}$-humidified atmosphere. Subsequently, cells were washed twice with medium and incubated at $37^{\circ} \mathrm{C}$ for $24 \mathrm{~h}$ in a $5 \% \mathrm{CO}_{2}$-humidified atmosphere with medium containing 0-16 mM VPA and/or 0-10 ng/ml TRAIL. A total of $2 \mathrm{mM}$ VPA was used in the single treatment group and in the VPA + TRAIL group, because TRAIL treatment to glioma cells started to significantly decrease glioma cells viability. After exposure to the various concentrations of VPA or TRAIL for $24 \mathrm{~h}$, the viable cell population was determined using MTT assay, with isopropanol:DMSO (9:1) used to dissolve the purple formazan. Then, the $570 \mathrm{~nm}$ wavelength was used to measure formazan.

For co-culture experiments, MSCs-TRAIL $\left(1 \times 10^{4}\right)$ were plated in Transwell inserts with $0.4-\mathrm{mm}$ pores (Corning, Inc.) and glioma cells $\left(5 \times 10^{4}\right)$ were seeded in the lower well of the 24-well plates. Following incubation for $24 \mathrm{~h}$ at $37^{\circ} \mathrm{C}$, apoptosis activity in glioma cells was measured using a caspase-Glo 3/7 assay (Promega Corporation). Briefly, an equal volume of caspase-3/7 detection reagent was added $24 \mathrm{~h}$ after combination treatment with VPA and TRAIL to glioma cells supernatants, and the mixtures were incubated at $37^{\circ} \mathrm{C}$ for $3 \mathrm{~h}$. Supernatants were obtained via centrifugation at room temperature and $2,012 \mathrm{xg}$ for $10 \mathrm{~min}$, and were stored at $-80^{\circ} \mathrm{C}$ for $\sim 7$ days. Each sample was measured with a SpectraMax L luminometer (Molecular Devices, LLC).

Western blotting. Western blot analysis was performed as described previous (24). Cells were lysed in protein extraction buffer (PRO-PREP ${ }^{\text {TM }}$ Protein Extraction Solution; Intron Biotechnology, Inc.). Protein concentrations were determined with the Bradford protein assay kit. The protein was subjected to $4-10 \%$ gradient gel by loading $15 \mu \mathrm{g}$ protein per lane, and western blot analysis was then performed. Proteins were transferred onto a nitrocellulose membrane (Invitrogen; Thermo Fisher Scientific, Inc.). Each blot was blocked using PBS containing 5\% skim milk and $0.05 \%$ Tween-20 at room temperature for $30 \mathrm{~min}$. The membranes were incubated with the appropriate primary antibodies against DR5 (1:500; R\&D Systems, Inc.; cat. no. MAB1540), caspase-3 (1:500; Cell Signaling Technology, Inc.; cat. no. 9662S), cleaved caspase-3 (1:500; Cell Signaling Technology, Inc.; cat. no. 9664S), caspase-8 (1:500; Cell Signaling Technology, Inc.; cat. no. 9746S), CXCR4 (1:500; Abcam; cat. no. ab2090) and $\beta$-actin (1:1,000; Sigma-Aldrich; Merck KGaA; cat. no. A1978) overnight at $4^{\circ} \mathrm{C}$. Subsequently, blots were incubated with secondary antibodies conjugated with horseradish peroxidase (1:1,000; Invitrogen; Thermo Fisher Scientific, Inc.; monoclonal cat. no. 32430, polyclonal cat. no. 32460) for $2 \mathrm{~h}$ at room temperature. The bands were detected using an enhanced chemiluminescence detection system (Cytiva). Each western blotting test was conducted on different parts using the same gel type and exposure.

Flow cytometric analysis - FACS. For the flow cytometric analysis of DRs, cells were harvested and incubated with phycoerythrin-conjugated anti human DR5 antibody (1:1,000; cat. no. AF631; R\&D Systems, Inc.), as described previously $(25,26)$. Briefly, cells $\left(2.5 \times 10^{5}\right)$ were stained with the antibody for $30 \mathrm{~min}$ at $4^{\circ} \mathrm{C}$. After washing with PBS, the expression of DR5 was analyzed via flow cytometry using a FACSVantage SE (Becton, Dickinson and Company). In addition, apoptosis was determined using an Annexin V and PI staining-based fluorescein isothiocyanate Annexin V 
Apoptosis Detection kit (BD Biosciences), according to the manufacturer's instructions, $24 \mathrm{~h}$ after treatment of glioma cells with VPA and TRAIL. The FASCanto 2 flow cytometer (BD Biosciences) and FlowJo v8.0.3 (FlowJo LLC) software were used for analysis for early + late apoptotic cells.

TRAIL expression using ELISA. The culture supernatant of MSC-TRAIL was analyzed using ELISA according to manufacturer's procedure (Human TRAIL/TNFSF10 Quantikine ELISA Kit; R\&D Systems, Inc.; cat. no. DTRL00). To investigate the persistence of transgene expression in vitro, BM-MSCs were seeded at a high density $\left(4 \times 10^{4}\right.$ per well of a 24-well plate). BM-MSCs were transduced with 50 multiplicities of infection of Ad-TRAIL in OPTI-MEM serum-free medium for $3 \mathrm{~h}$ in a $5 \% \mathrm{CO}_{2}$ humidified $37^{\circ} \mathrm{C}$ chamber. The virus-containing medium was removed and additionally incubated in low-serum medium (Eagle's DMEM containing $2 \% \mathrm{FBS}$ ) at $37^{\circ} \mathrm{C}$ for $\sim 40$ days. Culture supernatants were harvested and fresh medium was changed every 3 day. Then, secreted TRAIL was examined.

In vitro and in vivo migration assay. The migratory abilities of MSCs and MSCs-TRAIL were determined using Transwell plates (Costar; Corning, Inc.) that were 6.5-mm in diameter with $8-\mu \mathrm{m}$ pore filters. U87 and U138 $\left(5 \times 10^{5}\right)$ cells were incubated in serum-free medium (SFM) for $48 \mathrm{~h}$ and the resulting conditioned medium (CM) was used as a chemoattractant. MSCs or MSCs-TRAIL $\left(2 \times 10^{4}\right)$ cells were suspended in SFM and plated into the upper well, and $600 \mu \mathrm{l}$ SDF-1-containing SFM, CM or the CXCR4 antagonist AMD3100 was used to confirm the migration capacity of MSCs-TRAIL toward glioma cells was placed in the lower well of the Transwell plate. Following incubation for $4 \mathrm{~h}$ at $37^{\circ} \mathrm{C}$, cells that had not migrated from the upper side of the filter were scraped off with a cotton swab, and filters were stained with the Diff-Quik ${ }^{\mathrm{TM}}$ three-step stain set (Sysmex Corporation). The number of cells that migrated to the lower side of the filter was counted under a light microscope at x100 magnification in three randomly-selected fields.

For the in vivo migration assay, mice were used in this test $(n=6)$. For the in vivo migration assay, $1 \times 10^{5}$ red fluorescent PKH26 dye (Sigma-Aldrich; Merck KGaA)-labeled VPA-treated MSCs-TRAIL were mixed with an equal number of green fluorescent PKH67 dye (Sigma-Aldrich; Merck KGaA)-labeled MSCs-TRAIL. Mixed cells were implanted into the contralateral hemisphere at 2 weeks after glioma cell $\left(5 \times 10^{4}\right.$ cells in $\left.3 \mu \mathrm{l} \mathrm{PBS}\right)$ inoculation into the right striatum of the mouse brain. Migration toward the tumor was assessed via direct visualization at 7 days after mixed cell inoculation using an LSM 510 Meta confocal microscope (Carl Zeiss AG; magnification, $\mathrm{x} 100)$.

Intracranial glioma model and treatments. Nude mice (age, 6-8 weeks; weight, 20 g; Charles River Laboratories, Inc.) were used in accordance with institutional guidelines under approved by the Institutional Animal Care and Use Committee of The Catholic University of Korea (approval no. 2017-0211-05). The following housing conditions were used: Temperature $22 \pm 3^{\circ} \mathrm{C}$, relative humidity $60 \pm 15 \%, 12$-h light $/ 12$-h dark cycle and autonomous intake of food and water. Mice were divided into four groups ( $\mathrm{n}=28$, with 7/group): Tumor control group,
VPA-treated group, MSC-TRAIL-treated group and VPA with MSC-TRAIL-treated group. The intracranial xenograft mouse model of human glioma was established as previously described $(18,28,30)$. For intracranial implantation of human glioma cells in the brains of mice, mice were deeply anesthetized with ketamine-xylazine cocktail $(80 \mathrm{mg} / \mathrm{kg}$ ketamine, $10 \mathrm{mg} / \mathrm{kg}$ xylazine) (31) and then animals were stereotactically inoculated with 1x105 U87 cells (in $3 \mu \mathrm{l}$ PBS) into the right frontal lobe (2-mm lateral and 1-mm anterior to bregma, at 2.5-mm depth from the skull base) using a Hamilton syringe (Hamilton Company) and a microinfusion pump (Harvard Apparatus; Harvard Bioscience, Inc.) $(18,28,30)$.

For survival experiments, intracranial glioma-bearing mice were randomly divided into four groups after tumor implantation: i) Those treated with intratumoral injections of saline ( $3 \mu \mathrm{PBS}$ ); ii) received intraperitoneal injections of VPA (200 mg/kg); iii) received MSCs infected with Ad-TRAIL (MSCs-TRAIL; $\left.2 \times 10^{5}\right)$ via intracranial injection $(18,28,30)$; and iv) or combination therapy (VPA and MSCs-TRAIL). VPA was injected 1 day after MSC transplantation and continued every day for 7 days. Mice that exhibited rapid weight loss ( $\geq 10 \%$ in 3 days) or onset of significant neurological symptoms, such as seizures, impaired balance and hemiplegia, were considered in a moribund condition. Mice were euthanized via $\mathrm{CO}_{2}$ asphyxiation $(10-20 \% / \mathrm{min})$ when these symptoms were identified in the glioma mice model (32).

In vivo bioluminesence imaging analysis and evaluation of tumor region. In vivo bioluminesence imaging analysis was performed with survival analysis. For in vivo bioluminesence imaging, the animals were inoculated with U87-Luc and treated as aforementioned (30). To monitor animal condition, a Maestro device was used to assess the inhibition of tumor growth and monitor the condition of the mice every 4 days after tumor inoculation. The substrate of luciferase, D-luciferin (150 mg/kg; Xenogen Corporation), was delivered via intraperitoneally injection $15 \mathrm{~min}$ before direct visualization using the Maestro in vivo Imaging System (CRI, Inc.; http://www. cri-inc.com/products/maestro-2.asp). In brief, mice were anesthetized using gas mixtures of $1.5 \%$ isoflurane (dosage 2-5\% induction; $0.25-4 \%$ maintenance) (33) and then imaged. The biofluorescence signals (photons/sec) emitted from the mice were captured using a high-sensitivity charge-coupled device camera (PerkinElmer, Inc.) and analyzed using Maestro II software (CRI, Inc.). To evaluate the tumor region and in vivo fluorescence imaging analysis, mice were divided into four groups $(\mathrm{n}=12 ; 3 /$ group): Tumor control group, VPA-treated group, MSC-TRAIL-treated group and VPA with MSC-TRAIL-treated group. Brains from mice given therapeutic treatment were serially sectioned (thickness, $20 \mu \mathrm{m}$; obtained every $200 \mu \mathrm{m}$ into the tumor) at day 35 after tumor inoculation, then stained with hematoxylin and eosin (H\&E) and visualized directly with a Slide scanner (Pannoramic MIDI, 3D; Histech Ltd.). The brain was removed and the frontal part of the cerebrum was embedded in optimal cutting temperature compound (Tissue-Tek ${ }^{\circledR}$ O.C.T.) and snap-frozen in liquid nitrogen at $-196^{\circ} \mathrm{C}$. Embedded tissues cryosections were stained with Harris hematoxylin solution for $8 \mathrm{~min}$ and with eosin at room temperature for $30 \mathrm{sec}$ to $1 \mathrm{~min}$. Sections were mounted with xylene based mounting medium. 

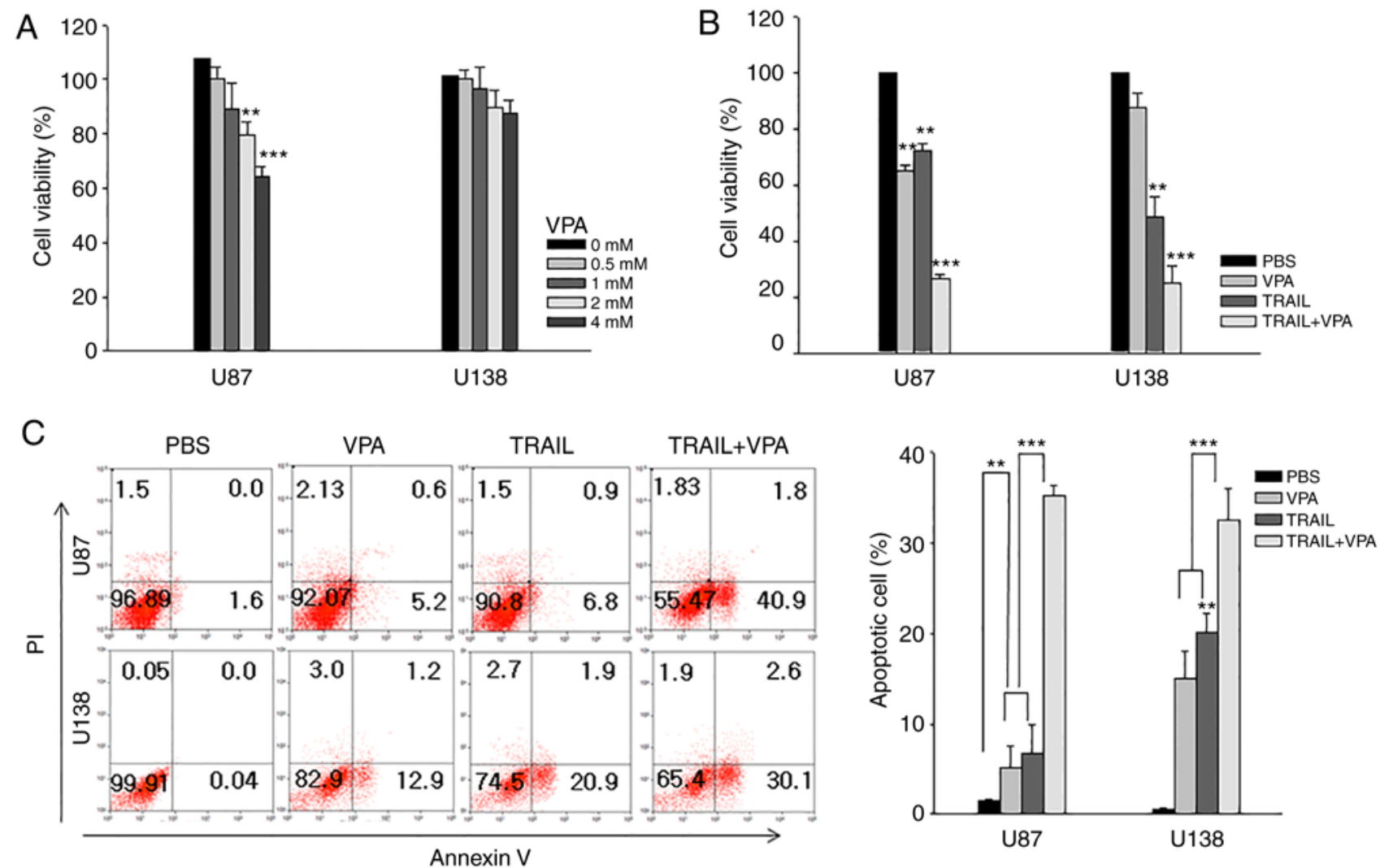

Figure 1. Cytotoxic effects of glioma cells using TRAIL and combined with VPA. (A) VPA sensitivity was achieved in glioma cells (U87 and U138). Glioma cells were treated with VPA in a dose-dependent manner $(0-4 \mathrm{mM})$ for $24 \mathrm{~h}$ and then analyzed with a MTT assay. ${ }^{* * *} \mathrm{P}<0.001$ vs. untreated control (one-way ANOVA).(B) To examine the effect of combined treatment, glioma cells were seeded in 96-well plates. The cytotoxic effect on glioma cells treated with VPA $(2 \mathrm{mM})$ and TRAIL $(10 \mathrm{ng} / \mathrm{ml})$ for $24 \mathrm{~h}$ was analyzed using a MTT assay. ${ }^{* * * *} \mathrm{P}<0.001 \mathrm{in}$ the comparison of alone treatment with untreated control; as compared with the treatment with VPA or TRAIL alone (one-way ANOVA with Bonferroni multiple comparison test). (C) Effect of VPA on TRAIL-induced apoptosis in glioma cells were evaluated via flow cytometry. Apoptosis was determined using Annexin V and PI staining at $24 \mathrm{~h}$ after treatment with VPA (2 mM) in the absence or presence of TRAIL $(10 \mathrm{ng} / \mathrm{ml})$. Data are presented as the mean $\pm \mathrm{SD}$. ${ }^{* *} \mathrm{P}<0.01$ in the comparison of alone treatment with untreated control; as compared with the treatment with VPA or TRAIL alone (one-way ANOVA with Bonferroni multiple comparison test). VPA, valproic acid; TRAIL, tumor necrosis factor-related apoptosis-inducing ligand.

Evaluation of apoptosis via TUNEL assay. Mouse brains were perfused with PBS, $4 \%$ paraformaldehyde and postfixed $4^{\circ} \mathrm{C}$ overnight. Fixed brains were embedded, snap frozen in liquid nitrogen at $-196^{\circ} \mathrm{C}$ and stored at $-70^{\circ} \mathrm{C}$ until use. TUNEL staining was performed using the TUNEL assay kit (Roche Diagnostics). Terminal deoxynucleotidyl transferase in reaction buffer (containing a fixed concentration of digoxigenin-labelled nucleotides) was applied to serial sections for $1 \mathrm{~h}$ at $37^{\circ} \mathrm{C}$, before the slides were placed in Stop/Wash buffer for $10 \mathrm{~min}$. Apoptotic cells were detected after incubation in the Cy3-conjugated streptavidin (Jackson ImmunoResearch Laboratories, Inc.) at 1:1,000 dilution for $30 \mathrm{~min}$ at room temperature. The slides were counterstained with DAPI (1:1,000; Sigma-Aldrich; Merck KGaA). Coverslips with Kaiser's glycerol gelatin (Merck KGaA; cat. no. 109242) mounting medium were used. Apoptotic cells were also measured via a computer using the MetaMorph (Molecular Devices).

Statistical analysis. Data are presented as the mean \pm SD, and were analyzed using SPSS 13.0 (SPSS, Inc.). Statistical differences between different test conditions were determined using unpaired t-test and post hoc Bonferroni test was used following one-way ANOVA analysis for multiple comparison.
The survival of glioma-bearing mice was analyzed using a log-rank test based on the Kaplan-Meier method. Experiments were repeated three times independently. $\mathrm{P}<0.05$ was considered to indicate a statistically significant difference.

\section{Results}

Combined treatment with VPA and TRAIL increases the cytotoxic effects. To assess whether VPA would be a useful reagent to increase sensitivity to TRAIL, glioma cells were treated with VPA. VPA increased cell death in glioma cells in a dose-dependent manner, as demonstrated by decreased cell viability (Fig. 1A). Combined treatment with VPA and TRAIL significantly induced cell death in glioma cells (Fig. 1B). To identify apoptotic cell death upon combined treatment in glioma cells, apoptotic cell populations were detected using FACS analysis. A significant increase in apoptotic cells was observed when glioma cells were exposed to combined treatment, compared with VPA or TRAIL alone (Fig. 1C).

VPA treatment activates TRAIL-induced apoptosis via DR5 upregulation. To evaluate if VPA increased TRAIL-induced apoptosis, it was examined whether VPA increased the number of TRAIL receptors. FACS analysis demonstrated that 

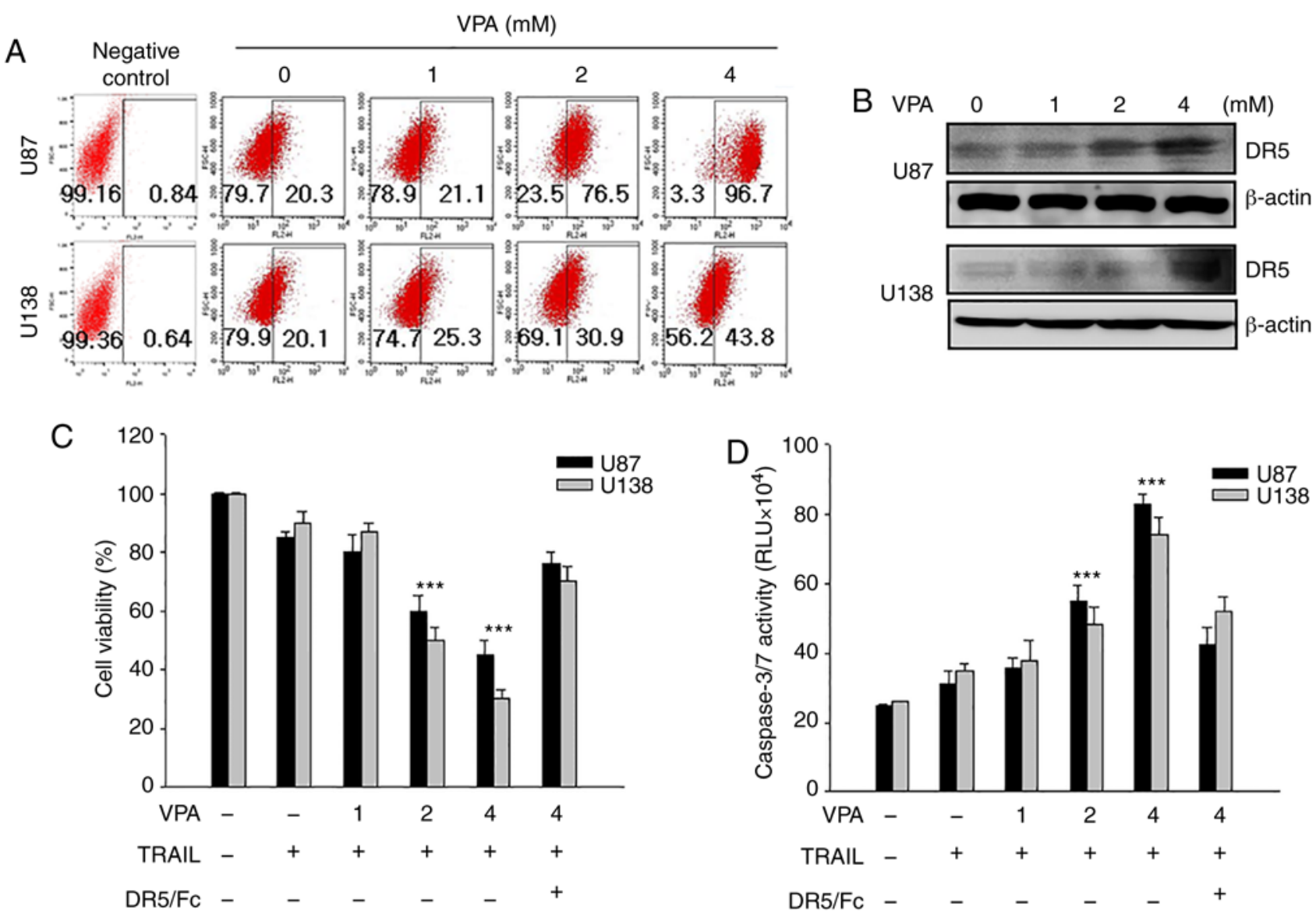

Figure 2. Enhancement of TRAIL-induced apoptosis by upregulation of DR5 and caspase activity with VPA treatment. (A) Glioma cells (U87 and U138) were treated with increasing doses of VPA ( $0-4 \mathrm{mM})$ for $24 \mathrm{~h}$ and DR5 expression was measured via flow cytometry. (B) Glioma cells were treated to VPA (0-4 mM) for $24 \mathrm{~h}$, and then total cell extracts were tested via western blotting with anti-DR5 antibody. $\beta$-actin expression was used as a loading control. (C) Glioma cells were treated with VPA $(0-4 \mathrm{mM})$ and TRAIL $(10 \mathrm{ng} / \mathrm{ml})$ with or without DR5/Fc chimera protein $(100 \mathrm{ng} / \mathrm{ml}$; to block DR5 activity), and then the viability of glioma cells was determined using a MTT assay. Data are presented as the mean $\pm \mathrm{SD}$. ${ }^{* * *} \mathrm{P}<0.001$ in the comparison of alone treatment with untreated control; as compared with the treatment with VPA or TRAIL alone (one-way ANOVA with Bonferroni multiple comparison test). (D) Glioma cells were treated with VPA (0-4 mM) or VPA combined with TRAIL $(10 \mathrm{ng} / \mathrm{ml})$ with or without DR5/Fc chimera protein $(100 \mathrm{ng} / \mathrm{ml})$ and tested with a caspase-Glo $3 / 7 \mathrm{kit}$ to analyze caspase activity. ${ }^{* * *} \mathrm{P}<0.001$ in the comparison of alone treatment with untreated control; as compared with the treatment with VPA or TRAIL alone (one-way ANOVA with Bonferroni multiple comparison test). DR, death receptor; VPA, valproic acid; TRAIL, tumor necrosis factor-related apoptosis-inducing ligand.

DR5 expression was increased in a dose-dependent manner in glioma cells (Fig. 2A), and western blot analysis also suggested a dose-dependent increase in DR5 expression after VPA treatment (Fig. 2B). To determine if apoptosis upon combined treatment was associated with DR5, DR5/Fc chimera proteins were used to block DR5 activity. In addition, it was investigated whether VPA-enhanced TRAIL-induced apoptosis was mediated via caspase activation. VPA and TRAIL combination decreased glioma cell viability and increased caspase-3/7 activity (Fig. 2C and D). Glioma cell death and caspase-3/7 activity were inhibited in the presence of DR $5 / \mathrm{Fc}$, indicating that apoptosis enhancement, caused by the combined treatment, may be associated with the specific interaction between TRAIL and DR5.

VPA increases the therapeutic effects of MSCs-TRAIL against glioma cells. Based on the effects of combined VPA and TRAIL treatment on glioma cells, MSCs were used as a TRAIL delivery vehicle to increase efficacy. VPA was added to MSCs or MSC-TRAIL to examine how to avoid the induction of MSC-TRAIL inhibition. In media containing various concentrations of VPA, the viability of MSCs or MSC-TRAIL was not changed (Fig. 3A). In addition, VPA did not affect the duration of TRAIL secretion in MSC-TRAIL (Fig. 3B). Glioma cells were treated with MSC-TRAIL and VPA at different doses, then the viability and the expression and cleavage of caspase-8, an initiator caspase, and caspase-3, a major effector caspase (34), were investigated. DR5/Fc was used to verify the specific interaction between DR5 and the combination treatment. Glioma cell death and cleavage of caspase- 8 and caspase-3 were increased upon combined treatment with VPA and MSC-TRAIL, while DR5/Fc blocked glioma cell death and caspase- 8 and caspase- 3 cleavage (Fig. 3C and D).

VPA treatment increases the migration of MSC-TRAIL via CXCR4 upregulation. The CXCR4/SDF-1 interaction serves a key role in MSC migration (35). The current study examined whether VPA treatment enhanced CXCR4 expression in MSC-TRAIL. It was found that CXCR4 expression was increased in MSCs and MSC-TRAIL following VPA treatment in a dose-dependent manner (0-4 mM) (Fig. 4A). Moreover, VPA increased MSC-TRAIL migration in a dose-dependent manner toward SDF-1, a ligand of CXCR4, but migration of VPA-treated MSC-TRAIL decreased after 

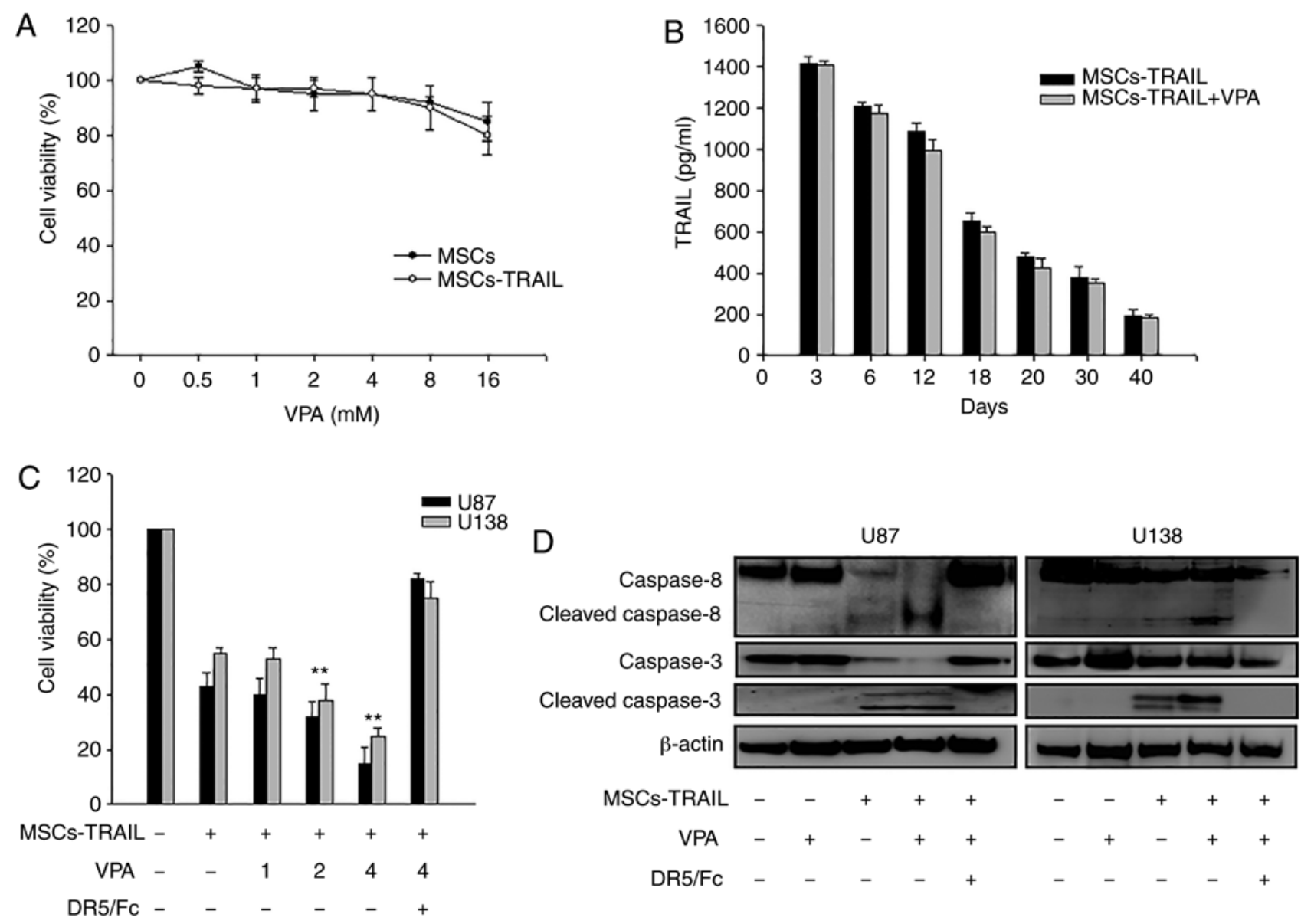

Figure 3. Effect of VPA treatment on MSCs-TRAIL. (A) MSCs and MSCs-TRAIL were treated with VPA (0-16 mM) and their viability was determined using a MTT assay. (B) After VPA ( $2 \mathrm{mM})$ was added to MSCs or MSCs-TRAIL, the concentration of secreted TRAIL in culture supernatant was analyzed via ELISA on the indicated day. (C) Glioma cells (U87 and U138) were treated with VPA (0-4 mM) with MSCs-TRAIL with or without DR5/Fc chimera protein $(100 \mathrm{ng} / \mathrm{ml})$, and then the viability of glioma cells was determined using a MTT assay. Data are presented as the mean $\pm \mathrm{SD}$. ${ }^{* *} \mathrm{P}<0.01$ in the comparison of alone treatment with untreated control; as compared with the treatment with VPA or MSCs-TRAIL alone (one-way ANOVA with Bonferroni multiple comparison test). (D) Glioma cells (U87 and U138) were treated with VPA (2 mM), MSCs-TRAIL or VPA and MSCs-TRAIL with or without DR5/Fc chimera protein $(100 \mathrm{ng} / \mathrm{ml})$. After $24 \mathrm{~h}$, total cell extracts were analyzed using western blotting with antibodies against caspase-8 (upper panel) and caspase-3 (bottom panel). The relative expression of each protein was normalized to $\beta$-actin. Each western blot test was conducted on different parts using same gel and exposure. VPA, valproic acid; TRAIL, tumor necrosis factor-related apoptosis-inducing ligand; DR, death receptor; MSCs-TRAIL, tumor necrosis factor-related apoptosis-inducing ligand-secreting human bone marrow-derived mesenchymal stem cells.

treatment with AMD3100, a CXCR4 antagonist (Fig. 4B). The migration of MSCs toward CM from glioma cells was examined. Combined treatment with VPA and MSC-TRAIL significantly enhanced the migration of MSCs toward CM from glioma cells (Fig. 4C). To assess the in vivo migratory capacity of VPA or MSC-TRAIL alone, PKH-26-labeled VPA-treated MSCs-TRAIL were injected together with PKH-67-labeled control MSCs-TRAIL into the contralateral hemisphere of tumors in a U-87MG glioma model. VPA increased the MSC-TRAIL migratory activity compared with MSC-TRAIL alone (Fig. 4D). These observations indicated that CXCR4/SDF-1 was involved in the migration of MSCs toward gliomas both in vitro and in vivo.

Combined treatment with VPA and MSCs-TRAIL enhances the therapeutic potential in intracranial xenografted models. The therapeutic effects of the combined treatment were investigated in an intracranial xenograft mouse model after inoculation with glioma cells. To observe tumor growth using in vivo bioluminescent imaging analysis, tumor-bearing mice with U87-Luc cells were established. A total of 7 days after tumor inoculation mice were treated with MSCs-TRAIL, and then VPA was injected into the animal model (Fig. 5A). H\&E staining (upper panel) demonstrated that the tumor size was smaller in mice treated with MSC-TRAIL and VPA in combination compared with in those treated with VPA or MSC-TRAIL alone (Fig. 5B). Moreover, the survival rate (bottom panel; Fig. 5B) was prolonged in mice that received the combination treatment compared with those treated with VPA or MSC-TRAIL alone and the control mice. At day 7 after U87 inoculation, tumors were injected intratumorally with MSCs-TRAIL. Moreover, VPA was injected 1 day after MSC transplantation and continued every day for 7 days ( $\mathrm{n}=7$ /group). Analysis of survival was conducted using a log-rank test based on the Kaplan-Meier method. The survival of VPA and MSCs-TRAIL treated mice was significantly improved survival compared with each of the other three groups $(\mathrm{P}<0.05$; Fig. 5B).

Tumor-bearing mice were established using U87-Luc cells, and tumor growth was measured. A notable decrease in bioluminescence was observed in mice treated with the 

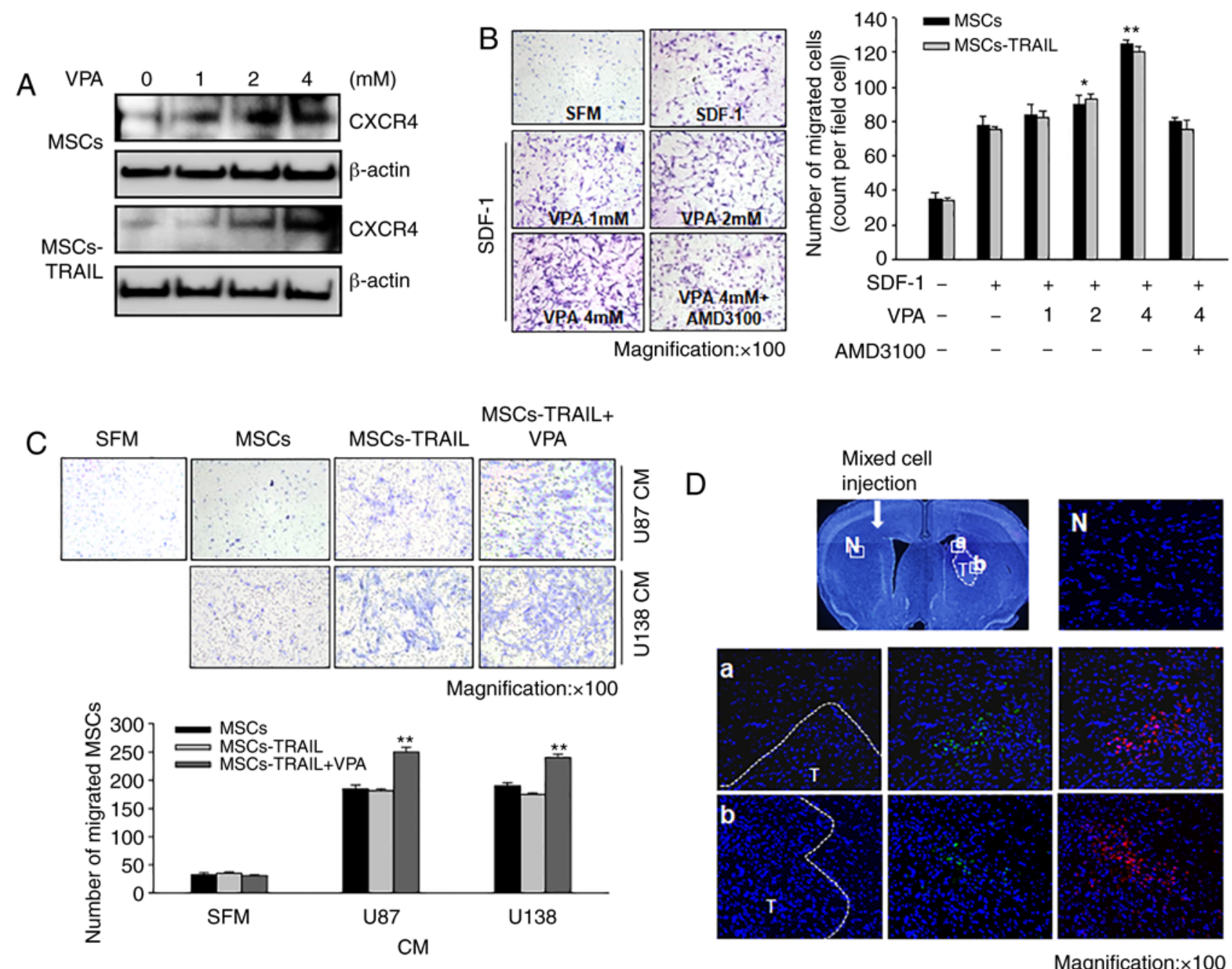

Figure 4. Increased migration ability of MSCs-TRAIL toward VPA-treated tumors and VPA-induced increase of CXCR4 expression in MSCs-TRAIL. (A) Upregulation of CXCR4 expression was identified via western blot analysis following addition of VPA to MSCs and MSCs-TRAIL culture medium. $\beta$-actin was used as a loading control. (B) Effect of treatment with SDF-1 and AMD3100 on the migration of MSCs-TRAIL was examined using a Transwell migration assay. Magnification, $x 100$. Data are presented as the mean \pm SD. ${ }^{*} \mathrm{P}<0.05$ and ${ }^{* *} \mathrm{P}<0.01$ vs. the treatment with SDF- 1 or VPA alone (one-way ANOVA with Bonferroni multiple comparison test). (C) Migratory ability of MSCs-TRAIL in response to conditioned medium from untreated or VPA-treated tumors was determined using a Transwell plate (8- $\mu \mathrm{m}$ pores). Representative photomicrographs of stained filters show migrated cells. Magnification, $\mathrm{x} 100$. Data are presented as the mean $\pm \mathrm{SD} .{ }^{* *} \mathrm{P}<0.01$ vs. treatment of MSCs-TRAIL or VPA (one-way ANOVA with Bonferroni multiple comparison test). (D) To measure the in vivo migration capacity of MSCs-TRAIL, PKH67(green)-labeled MSCs-TRAIL were injected together with PKH26(red)-labeled VPA-treated MSCs-TRAIL into the contralateral hemisphere of tumors in a glioma model. Nuclei were stained with DAPI (blue). Box a and b, Tumor area; Box N, Normal area; T, tumor area. Visualization was conducted using a confocal microscope (magnification, x100). Dotted line, tumor edge. VPA, valproic acid; TRAIL, tumor necrosis factor-related apoptosis-inducing ligand; DR, death receptor; MSCs-TRAIL, tumor necrosis factor-related apoptosis-inducing ligand-secreting human bone marrow-derived mesenchymal stem cells; SDF-1, stromal cell-derived factor-1; CXCR4, C-X-C chemokine receptor type 4; CM, conditioned medium; SFM, serum free medium.

combined treatment compared with those treated with VPA or MSC-TRAIL alone (Fig. 5C). Furthermore, TUNEL assay was performed to evaluate the MSC-TRAIL-induced apoptosis in VPA-treated glioma cells. Increased apoptotic activity was detected in MSC-TRAIL and VPA-treated glioma tumor regions compared with each treatment alone (Fig. 5D).

\section{Discussion}

The present study demonstrated that MSC-based TRAIL gene therapy, combined with VPA, was a more effective treatment against glioma compared with a single treatment. It was also identified that the therapeutic capacity of MSC-TRAIL was improved due to the increased number of DRs and the migratory ability of MSCs in response to VPA. Enhancement in MSC tropism appeared to be mediated by the increased CXCR4 release from MSCs in VPA-treated tumors. These findings suggested that VPA increased the anti-tumor activity of MSC-based TRAIL therapy by enhancing MSC tropism and TRAIL-induced apoptosis.

A HDACi, VPA, has been reported to enhance TRAIL sensitivity by inducing DR expression in several types of tumors, which can provide a positive anti-tumor effect against glioma by activating DR pathways (36-39). Although the current study did not demonstrated whether VPA with TRAIL affects healthy cells, the results suggested that VPA with TRAIL decreased viability of glioma cell lines (U87 and U138), as well as increased the apoptotic effect 
A

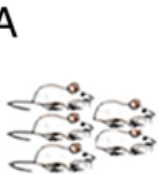

Nude mice

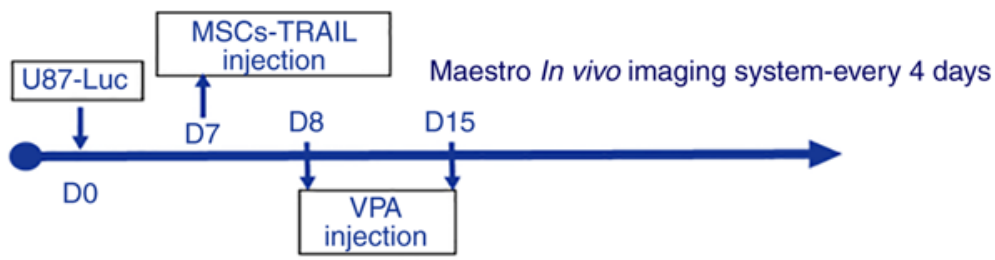

B
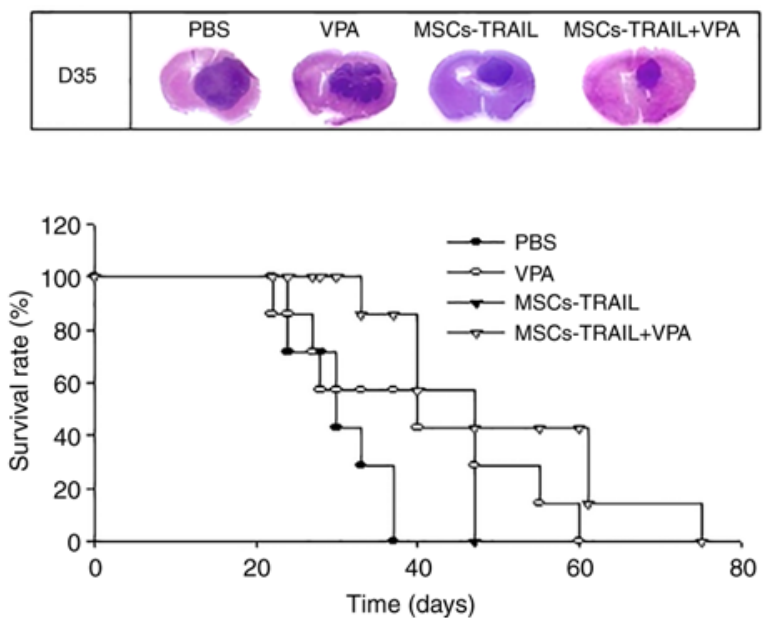
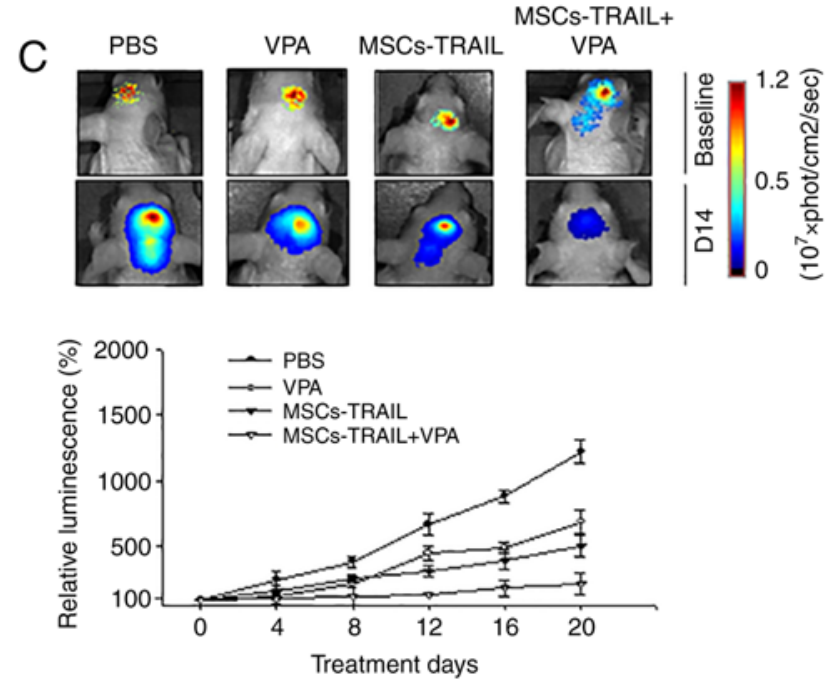

D

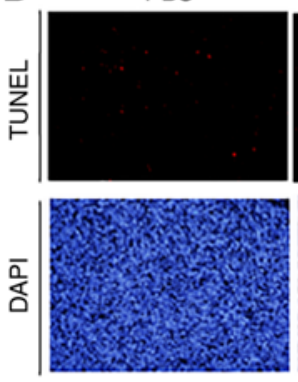

VPA

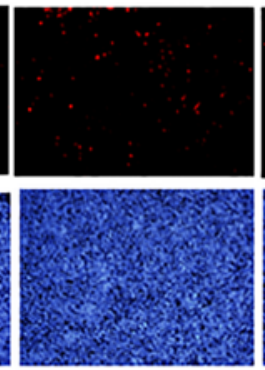

MSCS-TRAIL

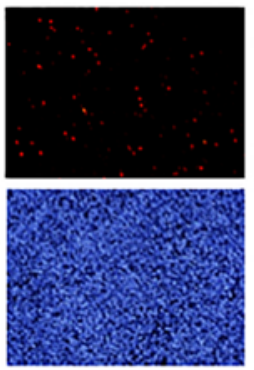

MSCS-TRAIL+VPA

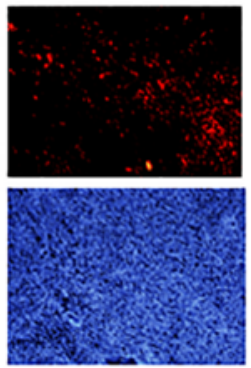

Magnification: $\times 100$

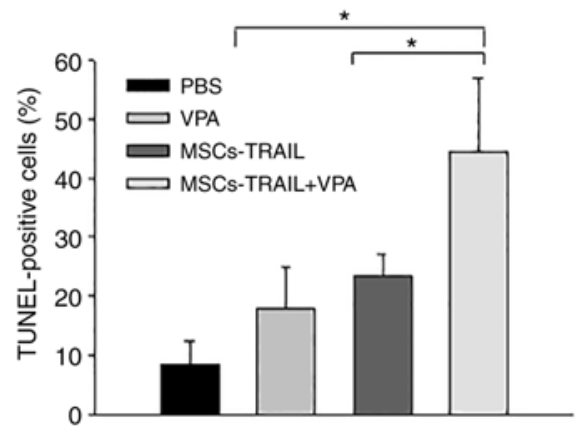

Figure 5. Effect of combined therapy with VPA and MSCs-TRAIL on tumor growth and survival in vivo. Tumor-bearing mice were treated with PBS, MSCs-TRAIL alone or VPA with MSCs-TRAIL to determine the effect on tumor growth in a intracranial glioma mice model. (A) Representation of animal groups and the schedule of animal experiment plan. (B) Representative images (magnification, x100) of hematoxylin and eosin staining from each treatment group, demonstrating the cell death in tumor mass at day 35 after tumor inoculation (upper panel). Survival rate of orthotopic xenograft mice was analyzed using a log-rank test based on the Kaplan-Meier method (bottom panel). (C) Bioluminescence images indicate tumor growth in orthotopic xenograft mice inoculated with U87-Luc cells after each treatment. (n=3/group). (D) Apoptosis in the treated groups was analyzed using TUNEL staining (red). Nuclei were stained with DAPI (blue). Visualization was performed using a confocal microscope at magnification, $\mathrm{x} 100$. Data are presented as the mean \pm SD. ${ }^{*} \mathrm{P}<0.05$ (one-way ANOVA with Bonferroni multiple comparison test). VPA, valproic acid; TRAIL, tumor necrosis factor-related apoptosis-inducing ligand; MSCs-TRAIL, tumor necrosis factor-related apoptosis-inducing ligand-secreting human bone marrow-derived mesenchymal stem cells; D, day.

in FACS and caspase $3 / 7$ activity. Thus, the present findings provide evidence for testing combination treatments in glioma cells, which may serve an important role in improving efficacy in glioma cell lines. The current study examined whether MSC-TRAIL treatment, combined with VPA, enhanced the potential therapeutic effect in glioma cells and an orthotopic xenograft mice model. Combined treatment with VPA and MSC-TRAIL significantly increased cytotoxicity in human glioma cells via TRAIL-induced apoptosis by upregulating DR5. However, treatment with individual agents weakly induced glioma cell cytotoxicity and DR5 upregulation. These results indicated that the VPA and MSC-TRAIL combination has the potential to increase cytotoxicity in glioma cells, overcoming the limitation of treatment with either alone.
In the present study, using MSCs as a delivery vehicle for TRAIL, along with VPA, it was observed that MSC viability and TRAIL secretion were affected. MSC viability or TRAIL release from engineered MSCs did not decrease significantly after exposure to TRAIL, even after combined treatment with VPA. These observations suggested that MSC-TRAIL released substantial quantities of TRAIL protein, so that the therapeutic gene was activated continuously and the effect was increased by VPA. The use of MSCs as delivery tools of anti-tumor agents has been reported for the treatment of gliomas $(40,41)$. The migration of MSCs toward various tumor sites is associated with the release of several cytokines (9). The enhanced migration of MSCs toward tumors makes them a potential combination strategy for anti-tumor therapy; however, accomplishing a 
sufficient number of tumor-targeted MSCs remains a problem. Recently, VPA was revealed to respond to SDF-1 and its receptor CXCR4, which are associated with bone marrow-derived cell migration, in a pathologic environment (42). The present study indicated that VPA treatment may be a potential a method to overcome the aforementioned-mentioned limitations of MSCs. The current results suggested that tumor treatment with VPA and MSC-TRAIL may enhance the migration of MSC-TRAIL toward glioma cells by enhancing CXCR4 expression in vitro. Furthermore, the in vivo observations demonstrated that VPA treatment with MSC-TRAIL increased migratory capacity toward tumor sites. Collectively, it was indicated that the increased expression of CXCR4 affected the migratory ability of MSCs; therefore, it may improve the therapeutic potential of MSC-based gene therapy.

In conclusion, the present results suggested that combining VPA and MSC-TRAIL may be an effective strategy in the treatment of malignant gliomas by enhancing apoptosis and migration. Therefore, the combination of VPA and MSC-TRAIL represents a potential therapeutic candidate for treating glioma. However, while the current study tested several possible means to increase TRAIL-induced apoptosis, the specific mechanism of sensitization were not identified. Thus, additional experiments are required to discover the specific mechanism of sensitization.

\section{Acknowledgements}

Not applicable.

\section{Funding}

This research was supported by the Ministry of Health and Welfare, Republic of Korea (grant no. HI18C2148) and the Basic Science Research Program through the National Research Foundation of Korea funded by the Ministry of Education (grant no. 2016R1D1A1B03931146).

\section{Availability of data and materials}

All data generated or evaluated during this study are included in this article.

\section{Authors' contributions}

SAP performed experiments described in the study and collected the data. HRH analyzed the results and wrote the manuscript. SA prepared the BM-MSCs and edited the manuscript. CHR and SSJ contributed to the conception and design of the study. All authors read and approved the manuscript.

\section{Ethics approval and consent to participate}

Animal experiments were approved by the Institutional Animal Care and Use Committee of The Catholic University of Korea (approval no. 2017-0211-05).

\section{Patient consent for publication}

Not applicable.

\section{Competing interests}

The authors declare that they have no competing interests.

\section{References}

1. Stupp R, Mason WP, van den Bent MJ, Weller M, Fisher B, Taphoorn MJ, Belanger K, Brandes AA, Marosi C, Bogdahn U, et al: Radiotherapy plus concomitant and adjuvant temozolomide for glioblastoma. N Engl J Med 352: 987-996, 2005.

2. Wilson TA, Karajannis MA and Harter DH: Glioblastoma multiforme: State of the art and future therapeutics. Surg Neurol Int 5: 64, 2014

3. Trivedi R and Mishra DP: Trailing TRAIL resistance: Novel targets for TRAIL sensitization in cancer cells. Front Oncol 5: 69, 2015.

4. Von Karstedt S, Montinaro A and Walczak H: Exploring the TRAILs less travelled: TRAIL in cancer biology and therapy. Nat Rev Cancer 17: 352-366, 2017.

5. Hodgkinson CP, Gomez JA, Mirotsou M and Dzau VJ: Genetic engineering of mesenchymal stem cells and its application in human disease therapy. Hum Gene Ther 21: 1513-1526, 2010.

6. Müller FJ, Snyder EY and Loring JF: Gene therapy: Can neural stem cells deliver? Nat Rev Neurosci 7: 75, 2006.

7. Cheng S, Nethi SK, Rathi S, Layek B and Prabha S: Engineered mesenchymal stem cells for targeting solid tumors: Therapeutic potential beyond regenerative therapy. J Pharmacol Exp Ther 370: 231-241, 2019.

8. Xu F and Zhu JH: Stem cells tropism for malignant gliomas. Neurosci Bull 23: 363-369, 2007.

9. Menon LG, Picinich S, Koneru R, Gao H, Lin SY, Koneru M, Mayer-Kuckuk P, Glod J and Banerjee D: Differential gene expression associated with migration of mesenchymal stem cells to conditioned medium from tumor cells or bone marrow cells. Stem Cells 25: 520-528, 2007.

10. Menon LG, Kelly K, Yang HW, Kim SK, Black PM and Carroll RS: Human bone marrow-derived mesenchymal stromal cells expressing S-TRAIL as a cellular delivery vehicle for human glioma therapy. Stem Cells 27: 2320-2330, 2009.

11. Kim SM, Kim DS, Jeong CH, Kim DH, Kim JH, Jeon HB, Kwon SJ, Jeun SS, Yang YS, Oh W and Chang JW: CXC chemokine receptor 1 enhances the ability of human umbilical cord blood-derived mesenchymal stem cells to migrate toward gliomas. Biochem Biophys Res Commun 407: 741-746, 2011.

12. Park SA, Ryu CH, Kim SM, Lim JY, Park SI, Jeong CH, Jun J, Oh JH, Park SH, Oh W and Jeun SS: CXCR4-transfected human umbilical cord blood-derived mesenchymal stem cells exhibit enhanced migratory capacity toward gliomas. Int J Oncol 38: 97-103, 2011.

13. Johnstone RW, Frew AJ and Smyth MJ: The TRAIL apoptotic pathway in cancer onset, progression and therapy. Nat Rev Cancer 8: 782-798, 2008

14. Dimberg LY, Anderson CK, Camidge R, Behbakht K, Thorburn A and Ford HL: On the TRAIL to successful cancer therapy? Predicting and counteracting resistance against TRAIL-based therapeutics. Oncogene 32: 1341-1350, 2013.

15. Zhang Land Fang B: Mechanisms of resistance to TRAIL-induced apoptosis in cancer. Cancer Gene Ther 12: 228-237, 2005.

16. Kim JY, Kim EH, Kim SU, Kwon TK and Choi KS: Capsaicin sensitizes malignant glioma cells to TRAIL-mediated apoptosis via DR5 upregulation and survivin downregulation. Carcinogenesis 31: 367-375, 2009.

17. Son YG, Kim EH, Kim JY, Kim SU, Kwon TK, Yoon AR, Yun CO and Choi KS: Silibinin sensitizes human glioma cells to TRAIL-mediated apoptosis via DR5 up-regulation and down-regulation of c-FLIP and survivin. Cancer Res 67: 8274-8284, 2007.

18. Kim SM, Oh JH, Park SA, Ryu CH, Lim JY, Kim DS, Chang JW, Oh W and Jeun SS: Irradiation enhances the tumor tropism and therapeutic potential of tumor necrosis factor-related apoptosisinducing ligand-secreting human umbilical cord blood-derived mesenchymal stem cells in glioma therapy. Stem Cells 28: 2217-2228, 2010

19. Fröscher W, Schulz H and Gugler R: Valproic acid in the treatment of epilepsy with special emphasis on serum level determination (author's transl). Fortschr Neurol Psychiatr Grenzgeb 46: 327-341, 1978 (In German). 
20. Cipriani A, Reid K, Young AH, Macritchie K and Geddes J: Valproic acid, valproate and divalproex in the maintenance treatment of bipolar disorder. Cochrane Database Syst Rev 10: CD003196, 2013.

21. Valiyaveettil D, Malik M, Joseph DM, Ahmed SF, Kothwal SA and Vijayasaradhi M: Effect of valproic acid on survival in glioblastoma: A prospective single-arm study. South Asian J Cancer 7: 159-162, 2018.

22. Searles CD, Slesinger PA and Singer HS: Effects of anticonvulsants on cholinergic and GABAergic properties in the neuronal cell clone NG108-15. Neurochem Res 13: 1007-1013, 1988.

23. Tarasenko N, Cutts SM, Phillips DR, Berkovitch-Luria G, Bardugo-Nissim E, Weitman M, Nudelman A and Rephaeli A: A novel valproic acid prodrug as an anticancer agent that enhances doxorubicin anticancer activity and protects normal cells against its toxicity in vitro and in vivo. Biochem Pharmacol 88: 158-168, 2014

24. Gotfryd K, Skladchikova G, Lepekhin EA, Berezin V, Bock E and Walmod PS: Cell type specific anti-cancer properties of valproic acid independent effects on HDAC activity and Erk12 phosphorylation. BMC Cancer 10: 383, 2010

25. $\mathrm{Xu} \mathrm{W}$, Parmigiani R and Marks P: Histone deacetylase inhibitors: Molecular mechanisms of action. Oncogene 26: 5541-5552, 2007

26. Göttlicher M, Minucci S, Zhu P, Krämer OH, Schimpf A Giavara S, Sleeman JP, Coco FL, Nervi C, Pelicci PG and Heinzel T: Valproic acid defines a novel class of HDAC inhibitors inducing differentiation of transformed cells. EMBO J 20 : 6969-6978, 2001.

27. Chateauvieux S, Morceau F, Dicato $M$ and Diederich $M$ : Molecular and therapeutic potential and toxicity of valproic acid. J Biomed Biotechnol 2010: 479364, 2010.

28. Kim SM, Lim JY, Park SI, Jeong CH, Oh JH, Jeong M, Oh W, Park SH, Sung YC and Jeun SS: Gene therapy using TRAIL-secreting human umbilical cord blood-derived mesenchymal stem cells against intracranial glioma. Cancer Res 68: 9614-9623, 2008.

29. Ryu CH, Park SA, Kim SM, Lim JY, Jeong CH, Jun JA, Oh JH Park SH, Oh WI and Jeun SS: Migration of human umbilical cord blood mesenchymal stem cells mediated by stromal cell-derived factor-1/CXCR4 axis via Akt, ERK, and p38 signal transduction pathways. Biochem Biophys Res Commun 398: 105-110, 2010.

30. Kim SM, Woo JS, Jeong CH, Ryu CH, Jang JD and Jeun SS: Potential application of temozolomide in mesenchymal stem cell-based TRAIL gene therapy against malignant glioma. Stem Cells Transl Med 3: 172-182, 2014.

31. Janssen CF, Maiello P, Wright MJ Jr, Kracinovsky KB and Newsome JT: Comparison of atipamezole with yohimbine for antagonism of xylazine in mice anesthetized with ketamine and xylazine. J Am Assoc Lab Anim Sci 56: 142-147, 2017.
32. Danneman PJ, Stein S and Walshaw SO: Humane and practical implications of using carbon dioxide mixed with oxygen for anesthesia or euthanasia of rats. Lab Anim Sci 47: 376-385, 1997.

33. Carpenter $\mathrm{J}$ and Chris M: Exotic animal formulary, 5th edition, pp468, 2017.

34. Mcllwain DR, Berger T and Mak TW: Caspase functions in cell death and disease. Cold Spring Harb Perspect Biol 5: a008656, 2013.

35. Naderi-Meshkin H, Bahrami AR, Bidkhori HR, Mirahmadi M and Ahmadiankia N: Strategies to improve homing of mesenchymal stem cells for greater efficacy in stem cell therapy. Cell Biol Int 39: 23-34, 2015 .

36. Rosato RR, Almenara JA, Dai Y and Grant S: Simultaneous activation of the intrinsic and extrinsic pathways by histone deacetylase (HDAC) inhibitors and tumor necrosis factor-related apoptosis-inducing ligand (TRAIL) synergistically induces mitochondrial damage and apoptosis in human leukemia cells. Mol Cancer Ther 2: 1273-1284, 2003.

37. Frew AJ, Lindemann RK, Martin BP, Clarke CJ, Sharkey J, Anthony DA, Banks K-M, Haynes NM, Gangatirkar P, Stanley K, et al: Combination therapy of established cancer using a histone deacetylase inhibitor and a TRAIL receptor agonist. Proc Natl Acad Sci USA 105: 11317-11322, 2008.

38. Bangert A, Cristofanon S, Eckhardt I, Abhari BA, Kolodziej S, Häcker S, Vellanki SHK, Lausen J, Debatin KM and Fulda S: Histone deacetylase inhibitors sensitize glioblastoma cells to TRAIL-induced apoptosis by c-myc-mediated downregulation of cFLIP. Oncogene 31: 4677-4688, 2012.

39. Bolden JE, Peart MJ and Johnstone RW: Anticancer activities of histone deacetylase inhibitors. Nat Rev Drug Discov 5: 769-784, 2006.

40. Nakamizo A, Marini F, Amano T, Khan A, Studeny M, Gumin J, Chen J, Hentschel S, Vecil G, Dembinski J, et al: Human bone marrow-derived mesenchymal stem cells in the treatment of gliomas. Cancer Res 65: 3307-3318, 2005.

41. Faderl S, O'Brien S, Pui CH, Stock W, Wetzler M, Hoelzer D and Kantarjian HM: Adult acute lymphoblastic leukemia: Concepts and strategies. Cancer 116: 1165-1176, 2010.

42. Marquez-Curtis LA and Janowska-Wieczorek A: Enhancing the migration ability of mesenchymal stromal cells by targeting the SDF-1/CXCR4 axis. Biomed Res Int 2013: 561098, 2013.

This work is licensed under a Creative Commons Attribution-NonCommercial-NoDerivatives 4.0 International (CC BY-NC-ND 4.0) License. 